\title{
Statin and Ezetimibe Combination Therapy Decreases Mean Platelet Volume Compared to Statin Monotherapy
}

\author{
Jun-beom Lee, ${ }^{\text {a }}$ Gyeong-Seon Kim, ${ }^{\text {b }}$ Han-na Cho ${ }^{c}$ \\ aDepartment of Neurology, Hong-lk Hospital, Seoul, Korea \\ bDepartment of Laboratory Medicine, Hong-Ik Hospital, Seoul, Korea \\ 'Department of Neurology, Gangnam Severance Hospital, Yonsei University College of Medicine, Seoul, Korea
}

\section{Dear Sir:}

When platelets are activated, they increase in size and change from a quiescent disc to a swollen sphere. Large platelets are more reactive and likely aggregate more than small ones. ${ }^{1}$ Mean platelet volume (MPV), an indicator of platelet size and activation, has been shown to predict ischemic stroke, myocardial infarction, and overall vascular mortality., ${ }^{2,3}$ The IMProved Reduction of Outcomes: Vytorin Efficacy International Trial (IMPROVEIT) study showed that ezetimibe added to statin therapy further decreases low density lipoprotein cholesterol (LDL-C) levels and prevented acute coronary syndromes. ${ }^{4}$ In this study, we investigated the lowering effect of LDL-C and MPV changes in patients who are on statin-ezetimibe combination therapy and those who receive statin monotherapy.

Patients with acute ischemic cerebral infarctions $(n=120)$ were divided into those who were on statin therapy $(n=60)$ and those on statin plus ezetimibe $(n=60)$. The statin group received either atorvastatin $(20 \mathrm{mg} ; \mathrm{n}=13)$ or rosuvastatin (10 mg; $n=48$ ). The statin plus ezetimibe group received ezetimibe (10 $\mathrm{mg}$ ) on top of the same dose of statin (atorvastatin plus ezetimibe, $n=14$; rosuvastatin plus ezetimibe, $n=47$ ). Student's $t$ tests showed that MPV and serum LDL-C levels were more significantly decreased (MPV: $P<0.001$; LDL-C: $P=0.017$ ) by statin plus ezetimibe combination therapy (MPV: from $7.39 \pm 1.04$ [5.8-9.2] to $6.04 \pm 0.58$ [4.9-7.3] fL; LDL-C: from $160.1 \pm 21.9$ [122-212] to $79.5 \pm 22.9$ [33-158] $\mathrm{mg} / \mathrm{dL}$ ) than statin monother- apy (MPV: from 7.47 \pm 0.74 [5.6-11.6] to 6.56 \pm 1.04 [4.9-9.2] fl; LDL-C: from 143.4 29.4 [52-238] to $95.6 \pm 21.7$ [41-150] mg/ $\mathrm{dL})$. Total cholesterol and triglycerides, but not high-density lipoprotein-cholesterol, were more significantly altered with combination therapy than with statin monotherapy (Table 1). A multivariate regression analysis showed that only $L D L-C$ in the lipid profile correlated significantly with the MPV change. A Pearson correlation analysis showed a positive correlation between the post-treatment MPV and post-treatment LDL-C levels ( $r=0.230$, $P=0.012$ ).

Statin has pleiotrophic effects and modulates platelet function by direct interactions with platelet membranes or by influencing signaling pathways, irrespective of the cholesterol-lowering effects. Aydin et al. reported that high doses of statin decrease MPV and LDL-C levels in patients with diabetes, but the changes in LDL-C and MPV did not correlate. ${ }^{5}$ Nevertheless, Marek et al. reported that MPV was correlated with small dense LDL-C. ${ }^{6}$ The possible mechanisms through which small dense LDL-C increases MPV include easier oxidation and glycation, which enhances the mobilization of arachidonic acid from phospholipids and decreases membrane fluidity. However, small dense-LDL-C measurements are time consuming and expensive in real life. In general, statin and ezetimibe induce reductions in all LDL-C subclasses. Ezetimibe blocks the critical mediator of cholesterol absorption, the Niemann-Pick C1-like 1 (NPC1L1) protein, on gastrointestinal tract epithelial cells as well as in hepatocytes, which causes them to absorb more LDL-C from the circulation and 
Table 1. Comparison of Lipid profile and mean platelet volume between statin and ezetimibe combination and statin monotherapy at baseline and post treatment

\begin{tabular}{|c|c|c|c|c|c|c|c|}
\hline & \multicolumn{3}{|c|}{ Statin plus ezetimibe } & \multicolumn{3}{|c|}{ Stain only } & \multirow{2}{*}{$P$-value } \\
\hline & At baseline & At follow up & Change (\%) & At baseline & At follow up & Change (\%) & \\
\hline $\mathrm{TC}(\mathrm{mg} / \mathrm{dL})$ & $234.5 \pm 36.8$ & $165.3 \pm 19.7$ & -29.5 & $231.5 \pm 37.4$ & $188.3 \pm 20.3$ & -19.6 & 0.039 \\
\hline LDL-C (mg/dL) & $160.1 \pm 21.9$ & $79.5 \pm 22.9$ & -50.4 & $143.4 \pm 29.4$ & $95.6 \pm 21.7$ & -33.4 & 0.017 \\
\hline $\mathrm{TG}(\mathrm{mg} / \mathrm{dL})$ & $189.1 \pm 22.4$ & $152.7 \pm 18.2$ & -19.2 & $183.5 \pm 21.9$ & $163.7 \pm 13.6$ & -10.7 & 0.042 \\
\hline $\mathrm{HDL}-\mathrm{C}(\mathrm{md} / \mathrm{dL})$ & $35.2 \pm 3.5$ & $44.7 \pm 1.9$ & 21.2 & $39.4 \pm 2.5$ & $47.8 \pm 5.8$ & 17.6 & 0.474 \\
\hline MPV (fL) & $7.39 \pm 1.04$ & $6.04 \pm 0.58$ & -18.3 & $7.47 \pm 0.74$ & $6.56 \pm 1.04$ & -12.2 & $<0.001$ \\
\hline
\end{tabular}

TC, Total cholesterol; LDL-C, Low density lipoprotein cholesterol; TG, Triglyceride; HDL-C, High density lipoprotein cholesterol; MPV, Mean platelet volume.

lower the circulating cholesterol level.

The previously proposed mechanisms of hypercholesterolemia in platelet activation are increased thromboxane $\mathrm{A} 2$ biosynthesis, inhibition of the platelet $\mathrm{Na}+/ \mathrm{H}+$ antiport, decreased platelet endogenous nitric oxide production, and diminished endothelial nitric oxide synthase (eNOS) protein expression. In addition, platelet activation by high LDL-C through platelet signaling pathways has been reported. ${ }^{8}$ The effects of ezetimibe monotherapy on the LDL subclass profile are controversial. Kasper et al. reported that ezetimibe increases small dense LDL-C in healthy men. ${ }^{9}$ In contrast, other studies have reported that ezetimibe decreases all LDL-C subclasses, including small dense LDL-C. ${ }^{10}$

Our results showed that MPV and LDL-C levels were more significantly decreased by statin and ezetimibe combination therapy than statin monotherapy and that their levels are closely correlated. Our data suggest that the lowering of LDL-C may causally related with MPV changes and support the "Lower LDLC, better outcome" findings of the IMPROVE-IT study. ${ }^{4}$

\section{References}

1. Martin JF, Trowbridge EA, Salmon G, Plumb J. The biological significance of platelet volume: its relationship to bleeding time, thromboxane B2 production and megakaryocyte nuclear DNA concentration. Thromb Res 1983;32:443-460.

2. Chu SG, Becker RC, Berger PB, Bhatt DL, Eikelboom JW, Konkle $B$, et al. Mean platelet volume as a predictor of cardiovascular risk: a systematic review and meta-analysis. J Thromb Haemost 2010;8:148-156.

3. Slavka G, Perkmann T, Haslacher H, Greisenegger S, Marsik C,

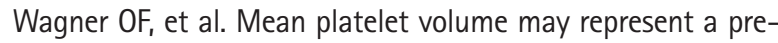
dictive parameter for overall vascular mortality and ischemic heart disease. Arterioscler Thromb Vasc Biol 2011;31:1215-1218.

4. Cannon CP, Blazing MA, Giugliano RP, McCagg A, White JA, Theroux $\mathrm{P}$, et al. Ezetimibe Added to Statin Therapy after Acute
Coronary Syndromes. N Engl J Med 2015;372:2387-2397.

5. Akyüz $A$, Akkoyun DÇ, Değirmenci $H$, Oran M. Rosuvastatin decreases mean platelet volume in patients with diabetes mellitus. Angiology 2016;67:116-120.

6. Marek K, David B, Peter K, Rachele C, Stanislav O, Luis R, et al. The effects of atorvastatin treatment on the mean platelet volume and red cell distribution width in patients with dyslipoproteinemia and comparison with plasma atherogenicity indicators-A pilot study. Clin Biochem 2015;48:557-561.

7. Alexandru N, Popov D, Dragan E, Andrei E, Georgescu A. Platelet activation in hypertension associated with hypercholesterolemia: effects of irbesartan. J Thromb Haemost 2011 ;9:173184.

8. Korporaal SJ, Akkerman JW. Platelet Activation by Low Density Lipoprotein and High Density Lipoprotein. Pathophysiol Haemos Thromb 2006;35:270-280.

9. Kaspar B, Manfredi R, Heiner KB, Giatgen AS, Wilhelm K, loanna GB. Ezetimibe alone or in combination with simvastatin increases small dense low-density lipoproteins in healthy men: a randomized trial. Eur Heart J 2010;31:1633-1639.

10. Kalogirou M, Tsimihodimos V, Gazi I, Filippatos T, Saougos V, Tselepis $A D$, et al. Effect of ezetimibe monotherapy on the concentration of lipoprotein subfractions in patients with primary dyslipidaemia. Curr Med Res Opin 2007;23:1169-1176.

Correspondence: Jun-beom Lee

Department of Neurology, Hong-lk Hospital, 225 Mokdong-ro, Yangchun-gu, Seoul 07937, Korea

Tel: +82-2-2600-0578, Fax: +82-2-2697-4605

E-mail: acupunct@hanmail.net

Received: May 15, 2016

Revised: July 21, 2016

Accepted: August 30, 2016

The authors have no financial conflicts of interest. 